\title{
Solar decimetric type III bursts in semi-closed magnetic field structures
}

\author{
H. Mészárosová ${ }^{1,2}$, M. Karlický2 ${ }^{2}$ H. S. Sawant ${ }^{1}$, F. C. R. Fernandes ${ }^{3}$, J. R. Cecatto ${ }^{1}$, and M. C. de Andrade ${ }^{1}$ \\ 1 National Space Research Institute (INPE), São José dos Campos, SP, Brazil \\ 2 Astronomical Institute of the Academy of Sciences of the Czech Republic, 25165 Ondřejov, Czech Republic \\ e-mail: hana@asu.cas.cz \\ 3 Institute of Research and Development (IP\&D - UNIVAP), São José dos Campos, SP, Brazil
}

Received 12 April 2007 / Accepted 1 April 2008

\begin{abstract}
Aims. We investigate statistically seventeen groups of solar type III bursts, observed in the frequency range 950-2500 MHz using the Brazilian Solar Spectroscope (BSS) during the period 1999-2002.

Methods. Using specially-developed Interactive Data Language (IDL) software called BSSView, the spectral characteristic properties of the bursts were investigated. For illustration a semi-closed magnetic field structure with trapped electron beams was modelled using the particle-in-cell model.

Results. Most of the type III bursts studied in this paper have starting frequencies in the range 1100-1400 MHz and their average bandwidth, half-power duration, and interval between adjacent bursts are $116 \pm 59 \mathrm{MHz}, 108 \pm 41 \mathrm{~ms}$, and $647 \pm 643 \mathrm{~ms}$, respectively. The number of bursts with negative frequency drift, which is on average $-914 \pm 684 \mathrm{MHz} \mathrm{s}^{-1}$, is greater than the number with positive frequency drift, which is on average $+807 \pm 675 \mathrm{MHz} \mathrm{s}^{-1}$. The type III bursts have power-law distribution function for their half-power duration $H\left(f_{\text {start }}\right)=4.79 \times 10^{8} f_{\text {start }}^{-2.145 \pm 0.015}$, bandwidth $B_{1}\left(f_{\text {start }}\right)=1.95 \times 10^{7} f_{\text {start }}^{-1.697 \pm 0.022}$ and $B_{2}\left(f_{\text {start }}\right)=7.07 \times 10^{5} f_{\text {start }}^{-1.154 \pm 0.015}$, and frequency drift $D\left(f_{\text {start }}\right)=0.43 f_{\text {start }}^{1.040 \pm 0.031}$. Most of the groups of type III bursts were also found to exhibit either positive or negative group frequency drifts of on average $+53 \pm 38 \mathrm{MHz} \mathrm{s}^{-1}$ and $-46 \pm 42 \mathrm{MHz} \mathrm{s}^{-1}$, respectively. The detailed statistical analysis suggests that in the frequency range studied there are two categories of type III bursts, the average parameters of which we provide in brackets for each type: a) numerous narrowband bursts (bandwidth $\sim 100 \mathrm{MHz})$ with small frequency drift $\left(\sim \pm 500 \mathrm{MHz} \mathrm{s}{ }^{-1}\right)$ in groups with visible group drift $\left(\sim \pm 50 \mathrm{MHz} \mathrm{s}^{-1}\right)$ and b) less numerous broader band bursts (bandwidth $\left.171 \mathrm{MHz}\right)$ with higher frequency drift $\left(\sim \pm 1800 \mathrm{MHz} \mathrm{s}^{-1}\right)$ in groups without group drift. The statistics of the parameters of these drifting groups are presented for the first time. The drifting groups of type III bursts are interpreted as due to electron beams trapped in moving plasmoids, which are semi-closed magnetic field structures.
\end{abstract}

Key words. Sun: radio radiation - Sun: corona - Sun: flares

\section{Introduction}

Decimetric bursts have been observed since 1960 and around 1973, for the first time, Skylab observations indicated that soft X-rays are generated in the solar atmosphere at $\sim 2 \times$ $10^{4} \mathrm{~km}$ above the photosphere, where densities are around $10^{9}-10^{10} \mathrm{~cm}^{-3}$ (Moore et al. 1980). X-ray studies by Ohyama \& Shibata (1998) suggested that the corresponding flare processes were occurring at the above mentioned densities, thereby indicating that acceleration of particles and/or heating was occurring there. These densities correspond to the plasma emission in the decimetric band $(\mathrm{dm})$ and hence have regenerated the interest in $\mathrm{dm}$-observations. These observations are important since solar radio emission observed in this band is considered to originate close to the region in which particle acceleration and energy release occur during solar flares (Tanuma \& Shibata 2005; Bárta \& Karlický 2005).

The flare-related radio emission in the $\mathrm{dm}$ range includes a wide variety of plasma-emission processes and is a useful tool for probing the associated energy release and electronacceleration processes. Isliker \& Benz (1994) analyzed type III bursts at 1-3 GHz, Cecatto et al. (2003) investigated highresolution time-profiles of decimetric type III bursts, and Sawant et al. (1994) reported investigations of microwave type III bursts with reverse slope drifts observed at $1600 \pm 100 \mathrm{MHz}$. Statistical studies of type III bursts were completed by Meléndez et al. (1999) at 1-3 GHz, and by Ma et al. (2002, 2005, and 2006) at $1-2,2.6-3.8$, and $0.65-0.80 \mathrm{GHz}$, respectively. These studies showed that type III bursts play an important role in the study of solar flares, revealing details of electron-acceleration processes and pinpointing the sites of energy release of flares in corona $(\mathrm{Li}$ et al. 2000; Lin et al. 2003). In contrast to dm type III bursts, metric type III bursts have larger bandwidth because they occur at more significant heights where the magnetic-field lines are open and their number is larger than that in closed magnetic structures (Kundu 1998). It is widely believed that when accelerated electrons escape along open field lines, broadband type III bursts, with negative drift rates, are generated (Poquérusse et al. 1995). In closed loops, the electron cyclotron maser (ECM), or coherent plasma-emission process, is driven mainly by plasma instabilities caused by magnetically-trapped electrons, which have precipitating electron-loss cone distributions that generate various types of fine structures. Electron beams travelling in descending and ascending legs of the magnetic structures can produce narrowband type III bursts with positive or negative drift rates (Aschwanden 2002; Benz 2004). Groups of dm type III bursts with negative group drifts were reported by Aschwanden \& Benz (1995). However, our investigations suggest that there are groups 
Table 1. Basic characteristics of the studied groups of type III bursts.

\begin{tabular}{|c|c|c|c|c|c|c|c|c|c|c|c|c|}
\hline \multirow[b]{2}{*}{ No. } & \multirow[b]{2}{*}{ Day } & \multicolumn{2}{|c|}{ Radio } & \multicolumn{4}{|c|}{ GOES } & \multicolumn{5}{|c|}{$\mathrm{H}_{\alpha}$} \\
\hline & & $\begin{array}{l}\text { Start } \\
\text { [UT] }\end{array}$ & $\begin{array}{l}\text { End } \\
{[\mathrm{UT}]}\end{array}$ & $\begin{array}{l}\text { Start } \\
{[\mathrm{UT}]}\end{array}$ & $\begin{array}{l}\operatorname{Max} \\
{[\mathrm{UT}]}\end{array}$ & $\begin{array}{c}\text { End } \\
{[\mathrm{UT}]}\end{array}$ & $\begin{array}{l}\text { X-ray } \\
\text { Imp. }\end{array}$ & $\begin{array}{l}\text { Start } \\
{[\mathrm{UT}]}\end{array}$ & $\begin{array}{l}\operatorname{Max} \\
{[\mathrm{UT}]}\end{array}$ & $\begin{array}{l}\text { End } \\
{[\mathrm{UT}]}\end{array}$ & $\begin{array}{l}\text { Optic. } \\
\text { Imp. }\end{array}$ & $\begin{array}{r}\text { Position, NOAA } \\
\text { AR \# }\end{array}$ \\
\hline 1 & Jun. 27, 1999 & $17: 21$ & $17: 22$ & & & & & & & & & \\
\hline 2 & Jun. 28, 1999 & $12: 21$ & $12: 22$ & $13: 13$ & $12: 25$ & $12: 17$ & C1.6 & $12: 13$ & $12: 25$ & $12: 18$ & SF & S27 E54 8611 \\
\hline 3 & Jul. 19, 1999 & $15: 08$ & $15: 09$ & & & & & $14: 59$ & $14: 59$ & $15: 03$ & SF & N20 E56 8636 \\
\hline 4 & Sep. 13, 1999 & $17: 43$ & $17: 44$ & $17: 23$ & $17: 32$ & $17: 38$ & $\mathrm{C} 2.1$ & $17: 35$ & $17: 37$ & $17: 40$ & SF & N17 E75 8700 \\
\hline 5 & Nov. 26, 1999 & $16: 26$ & $16: 28$ & & & & & & & & & \\
\hline 6 & May 08, 2000 & $15: 35$ & $15: 36$ & & & & & $15: 29$ & $15: 29$ & $15: 45$ & SF & N14 E53 8990 \\
\hline 7 & Jun. 06, 2000 & $15: 06$ & $15: 07$ & $14: 58$ & $15: 25$ & $15: 40$ & $\mathrm{X} 2.3$ & $15: 03$ & $15: 28$ & $15: 40$ & $2 \mathrm{~B}$ & N20 E13 9026 \\
\hline 8 & Jul. 13, 2000 & $14: 48$ & $14: 49$ & & & & & $14: 24$ & $14: 48$ & $14: 53$ & SF & N20 W74 9070 \\
\hline 9 & Sep. 20, 2000A & $12: 07$ & $12: 08$ & $11: 51$ & $11: 56$ & $12: 01$ & C 3.4 & $11: 52$ & $11: 56$ & $12: 10$ & SF & N05 E52 9169 \\
\hline 10 & Sep. $20,2000 B$ & $15: 51$ & $15: 55$ & $15: 19$ & $15: 31$ & $15: 42$ & C 3.1 & $15: 14$ & $15: 21$ & $15: 45$ & SF & N09 E41 9169 \\
\hline 11 & Sep. $20,2000 C$ & $17: 05$ & $17: 06$ & & & & & & & & & \\
\hline 12 & Sep. 21, 2000 & $18: 22$ & $18: 23$ & & & & & & & & & \\
\hline 13 & Sep. 26, 2000 & $12: 33$ & $12: 34$ & $12: 28$ & $12: 31$ & $12: 41$ & C4.3 & $12: 32$ & $12: 33$ & $12: 40$ & SF & S15 E59 9173 \\
\hline 14 & Aug. 22, 2001 & $15: 13$ & $15: 15$ & $15: 02$ & $15: 06$ & $15: 10$ & C3.7 & $15: 02$ & 15:04 & $15: 23$ & SF & S17 E77 9591 \\
\hline 15 & Sep. 13, 2001A & 13:04 & $13: 06$ & $13: 03$ & $13: 06$ & 13:07 & $\mathrm{C} 2.4$ & $13: 06$ & 13:07 & 13:07 & SF & S15 W10 9610 \\
\hline 16 & Sep. 13, 2001B & $16: 06$ & $16: 08$ & $15: 57$ & $16: 02$ & $16: 04$ & C7.0 & $16: 00$ & $16: 03$ & $16: 10$ & SF & S15 W10 9610 \\
\hline 17 & Jul. 11, 2002 & $14: 48$ & $14: 49$ & $14: 44$ & $14: 51$ & $14: 57$ & M5.8 & $14: 44$ & $14: 53$ & $15: 13$ & $2 \mathrm{~N}$ & W21 E58 10030 \\
\hline
\end{tabular}

of type III bursts that exhibit group drift, and also groups of bursts without group drift, even if individual type III bursts in the group have either positive or negative frequency drifts.

We investigate the group frequency drift and various characteristic properties of individual type III bursts such as half-power duration, bandwidth, frequency drift, and the intervals between bursts, in addition to possible relations between these properties.

The layout of the paper is as follows. In Sect. 2, we describe the instrument and the observations. We then study the characteristic parameters of the individual type III bursts and their groups in Sect. 3. Discussion of our results and conclusions is provided in Sect. 4.

\section{Instrument and observational data}

The frequency range of the Brazilian Solar Spectroscope (BSS) is $950-2500 \mathrm{MHz}$ with variable time and frequency resolution between 10-1000 ms and 1-10 MHz, respectively. The sensitivity is, in general, 1 solar flux unit (SFU) or 10000 Jy (Sawant et al. 2001). The BSS recorded frequency range at the time of observations depends on the frequency resolution.

During the years 1999-2002, 262 type III bursts were observed by the BSS instrument and these were separated into 17 groups, which are listed in Table 1. Most groups (13) were detected at frequencies $f<2000 \mathrm{MHz}$ and only four groups were detected at frequencies $f>2000 \mathrm{MHz}$. Seven groups were recorded with time and frequency resolutions, of 50 or $100 \mathrm{~ms}$ and $10 \mathrm{MHz}$, respectively, in the $1000 \mathrm{MHz}$ recorded range. In seven cases, the time and the frequency resolution was $50 \mathrm{~ms}$ and $5 \mathrm{MHz}$, respectively, and the recorded range was $500 \mathrm{MHz}$. In three cases the time and the frequency resolution was $20 \mathrm{~ms}$ and $4 \mathrm{MHz}$, respectively, and the recorded range was $200 \mathrm{MHz}$. The numbers of considered bursts in the recorded ranges are presented in Table 2 where the burst groups are divided into two parts. The first part, Part 1, contains 11 groups of type III bursts with group frequency drift. Most of the bursts in this part (171 of 201) are observed within the recorded frequency range and only 30 bursts of them are escaping out of the range. The second part of bursts, Part 2, consists of 6 groups of type III bursts without group frequency drift; 26 bursts, out of 61, are observed within the recorded range, and most bursts in this part (35 bursts) escape this frequency range.
Table 2. Recorded frequency range at the time of observations (RFR) and number of observed III type bursts ( $\mathrm{D}=$ drifting bursts, ID = bursts with immeasurable drift, $S=$ sum). Part 1 and Part 2 presents groups of type III bursts with and without global group drift, respectively.

\begin{tabular}{|c|c|c|c|c|c|c|c|c|}
\hline \multirow{3}{*}{$\begin{array}{c}\text { RFR } \\
{[\mathrm{MHz}]}\end{array}$} & \multirow{3}{*}{$\begin{array}{l}\text { Number } \\
\text { of } \\
\text { groups }\end{array}$} & \multicolumn{7}{|c|}{ Number of observed bursts } \\
\hline & & \multirow{2}{*}{$\begin{array}{c}\text { total } \\
\mathrm{S} 3+\mathrm{S} 4\end{array}$} & \multicolumn{3}{|c|}{ within RFR } & \multicolumn{3}{|c|}{ escaping RFR } \\
\hline & & & $\mathrm{D}$ & ID & S3 & $\mathrm{D}$ & ID & S4 \\
\hline & Part 1 & & & & & & & \\
\hline 1000 & 6 & 108 & 41 & 56 & 97 & 1 & 10 & 11 \\
\hline 500 & 4 & 79 & 53 & 11 & 64 & 3 & 12 & 15 \\
\hline 200 & 1 & 14 & 6 & 4 & 10 & 3 & 1 & 4 \\
\hline S1 & 11 & 201 & 100 & 71 & 171 & 7 & 23 & 30 \\
\hline & Part 2 & & & & & & & \\
\hline 1000 & 1 & 9 & 8 & 1 & 9 & - & - & - \\
\hline 500 & 3 & 20 & 8 & 1 & 9 & 9 & 2 & 11 \\
\hline 200 & 2 & 32 & 4 & 4 & 8 & 8 & 16 & 24 \\
\hline S2 & 6 & 61 & 20 & 6 & 26 & 17 & 18 & 35 \\
\hline $\begin{array}{c}\text { total } \\
\mathrm{S} 1+\mathrm{S} 2\end{array}$ & 17 & 262 & 120 & 77 & 197 & 24 & 41 & 65 \\
\hline
\end{tabular}

\subsection{Relationship between type III bursts and X-ray and $\mathrm{H} \alpha$ flares}

Among 17 groups (Table 1) of type III bursts in the present paper, there are 10 and 13 groups associated with GOES $\mathrm{X}$-ray events and $\mathrm{H} \alpha$ flares, respectively. Four of the type III bursts groups associated with GOES events (June 28, 1999, June 6, 2000, September 13, 2001A and July 11, 2002) occur at the impulsive phase and six of them (importance C) during the flare declining phase.

\section{Statistics and data analysis}

We considered 262 individual type III bursts for detailed statistical analysis of their individual frequency drift, bandwidth and half-power duration. We emphasize that we completed, for the first time, a statistical analysis of groups of bursts that exhibit group frequency drift. We considered (see Table 2): (i) 120 drifting bursts observed within the recorded frequency range (Sects. 3.1-3.3), (ii) 24 drifting bursts that escape the recorded range (Sect. 3.2), and (iii) 77 bursts with immeasurable 


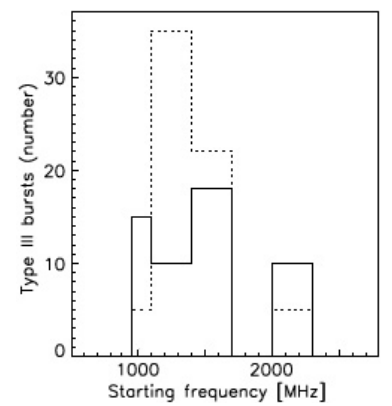

Fig. 1. Number of type III bursts versus starting frequency; solid and dashed lines represent the bursts with positive and negative frequency drift, respectively.

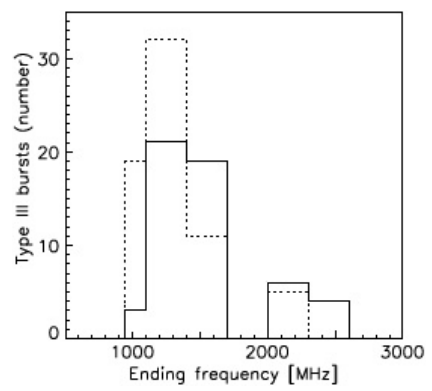

Fig. 2. Number of type III bursts versus ending frequency; solid and dashed lines represent bursts with positive and negative frequency drift, respectively.

drift observed within the recorded range (Sect. 3.2). The groups of type III bursts with and without group frequency drift (Part 1 and Part 2 in Table 2) are studied in Sects. 3.4 and 3.5, respectively.

\subsection{Distribution and half-power duration}

Of the 197 individual type III radio bursts observed within the recorded range (Table 2), 53 bursts show positive drift (from lower to higher frequencies) and 67 bursts show negative drifts (from higher to lower frequencies). For 77 bursts, the drift rate could not be determined. The starting frequency distribution (Fig. 1) shows 120 (53 and 67) drifting bursts in the range of 950-2500 MHz. Most of them (45) occur in the range $1100-1400 \mathrm{MHz}$ and 15 in the range $2000-2300 \mathrm{MHz}$. The ending frequency distribution (Fig. 2) shows 120 bursts in a similar frequency range. Most (53) occur in the range $1100-1400 \mathrm{MHz}$ and 11 in the range 2000-2300 MHz. Bursts with positive and negative drifts are not equal in number, in particular for the starting frequency distribution, for which there are 10 bursts with positive drift and 35 bursts with negative drift in the $1100-1400 \mathrm{MHz}$ range.

From the data analysis, we found that the half-power duration of 120 type III drifting bursts lasts between 29 and $382 \mathrm{~ms}$, with an average duration of $108 \pm 41 \mathrm{~ms}$. Most occur in the range 50-150 ms (Fig. 3). The half-power duration was found to decrease with increasing frequency (Table 3 ). We studied the distribution of the half-power duration with respect to the starting frequency of the bursts (Fig. 4). This distribution is fitted (solid line in Fig. 4) by the function $H\left(f_{\text {start }}\right)=4.79 \times 10^{8} f_{\text {start }}^{-2.145 \pm 0.015}$, where $H\left(f_{\text {start }}\right)$ is the half-power duration [ms] and $f_{\text {start }}$ is the starting frequency $[\mathrm{MHz}]$ of the type III bursts.

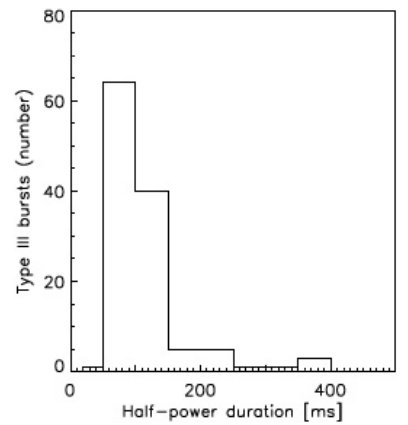

Fig. 3. Histogram of the distribution of half-power duration of type III bursts.

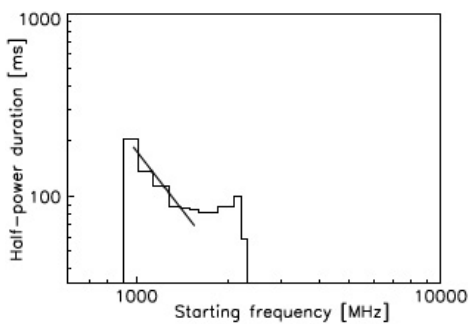

Fig. 4. Distribution of the half-power duration versus starting frequency of the bursts. This distribution is fitted by the solid line.

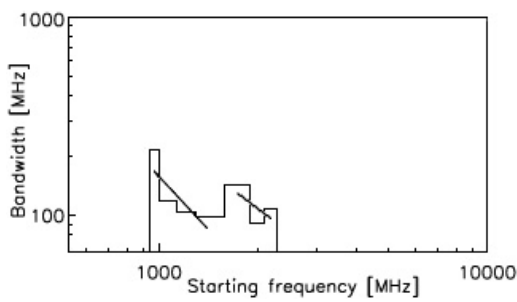

Fig. 5. Two distributions of the frequency bandwidth versus starting frequencies (about 1000 and $2000 \mathrm{MHz}$ ) of the bursts. These distributions are fitted by the solid lines.

\subsection{Frequency bandwidth and drift rates}

The bandwidth of 120 type III bursts with measurable frequency drift (61\% of the total 197 bursts), within the recorded range (Table 2), is between 40 and $468 \mathrm{MHz}$, with an average value of $116 \pm 59 \mathrm{MHz}$. The average frequency bandwidth (Table 3) decreases with increasing frequency. We studied the distribution (Fig. 5) of the frequency bandwidth with respect to the starting frequency of the bursts. This distribution is fitted in two frequency ranges (around $1000 \mathrm{MHz}$ and $2000 \mathrm{MHz}-$ solid lines in Fig. 5), by $B_{1}\left(f_{\text {start }}\right)=1.95 \times 10^{7} f_{\text {start }}^{-1.697 \pm 0.022}$, and $B_{2}\left(f_{\text {start }}\right)=7.07 \times 10^{5} f_{\text {start }}^{-1.154 \pm 0.015}$, respectively, where $B\left(f_{\text {start }}\right)$ is the frequency bandwidth [MHz], and $f_{\text {start }}$ is the starting frequency $[\mathrm{MHz}]$ of the type III bursts. We compare the split of the distribution (Fig. 5) with that in Figs. 1 and 2. The bandwidth of 77 type III bursts with immeasurable drift (39\% of 197 bursts) is between 21 and $189 \mathrm{MHz}$, with an average of $77 \pm$ $28 \mathrm{MHz}$. Thus, these bursts show narrower bands than the drifting ones.

We analyzed the individual frequency drift for 120 type III bursts within the recorded range (Table 2). Among them there are 53 bursts with positive drift and 67 with negative drift. The frequency drift of bursts with positive drift ranges from 98 to $2627 \mathrm{MHz} \mathrm{s}^{-1}$, with an average of $+505 \pm 346 \mathrm{MHz} \mathrm{s}^{-1}$, while for negative-drift bursts, the range is from -92 to 
Table 3. Average values of half-power duration, frequency bandwidth, interval between bursts, and frequency drift of the 120 drifting type III bursts (Table 2), with respect to their starting frequency.

\begin{tabular}{ccccc}
\hline $\begin{array}{c}\text { Starting frequency } \\
{[\mathrm{MHz}]}\end{array}$ & $\begin{array}{c}\text { Half-power duration } \\
{[\mathrm{ms}]}\end{array}$ & $\begin{array}{c}\text { Bandwidth } \\
{[\mathrm{MHz}]}\end{array}$ & $\begin{array}{c}\text { Interval between bursts } \\
{[\mathrm{ms}]}\end{array}$ & $\begin{array}{c}\text { Frequency drift } \\
{\left[\mathrm{MHz} \mathrm{s}^{-1}\right]}\end{array}$ \\
\hline$<1000$ & 206 & 214 & 592 & $|611|$ \\
$1000-2000$ & 103 & 110 & 651 & $|627|$ \\
$>2000$ & 93 & 104 & 642 & $|901|$ \\
\hline
\end{tabular}

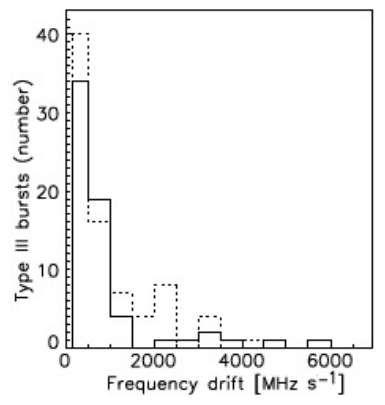

Fig. 6. Histogram of the distribution of the frequency drift for the selected bursts. Solid and dashed lines represent positive and negative drifts, respectively.

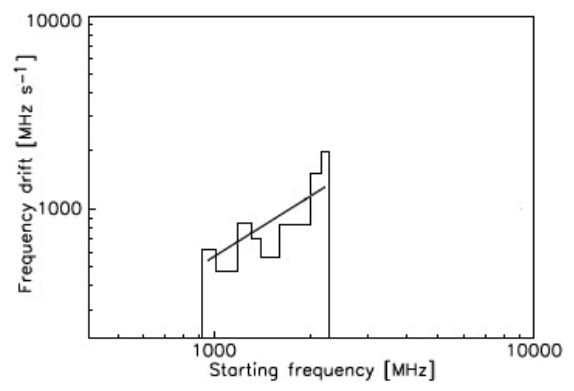

Fig. 7. Distribution of the frequency drift (absolute values) with respect to starting frequency of the bursts. This distribution is fitted by the solid line.

$-3441 \mathrm{MHz} \mathrm{s}^{-1}$, with an average $-783 \pm 572 \mathrm{MHz} \mathrm{s}^{-1}$. Figure 6 shows the number of type III bursts with respect to positive and negative drift. It can be seen that most bursts have frequency drift in the range $100-1000 \mathrm{MHz} \mathrm{s}^{-1}$. From Table 3, it is apparent that the average frequency drift increases with increasing frequency.

We measured the frequency drift of 24 drifting bursts that escaped the recorded range (Table 2). Among them, there are 11 bursts with positive drift and 13 with negative drift. The frequency drift of these bursts with positive drift ranges from 336 to $5528 \mathrm{MHz} \mathrm{s}^{-1}$, with an average $+2264 \pm 1648 \mathrm{MHz} \mathrm{s}^{-1}$, while for the negative-drifting bursts, the range is from -145 to $-4417 \mathrm{MHz} \mathrm{s}^{-1}$, with an average $-1593 \pm 921 \mathrm{MHz} \mathrm{s}^{-1}$. For all $144(120+24)$ drifting bursts (Table 2), the average value for the positive and negative drift is $+807 \pm 675 \mathrm{MHz} \mathrm{s}^{-1}$ (64 bursts) and $-914 \pm 684 \mathrm{MHz} \mathrm{s}^{-1}$ (80 bursts), respectively. Thus, there are more bursts with negative drift than those with positive drift, in general, and the bursts escaping the recorded range have significantly higher frequency drift than the bursts measured within the recorded range. Figure 7 shows the distribution of the frequency drift with respect to the starting frequency of the 144 bursts. This distribution is fitted by the solid line in Fig. 7, which is given by $D\left(f_{\text {start }}\right)=0.43 f_{\text {start }}^{1.040 \pm 0.031}$, where $D\left(f_{\text {start }}\right)$ is the frequency drift $\left[\mathrm{MHz} \mathrm{s}^{-1}\right]$, and $f_{\text {start }}$ is the starting frequency $[\mathrm{MHz}]$ of the type III bursts.

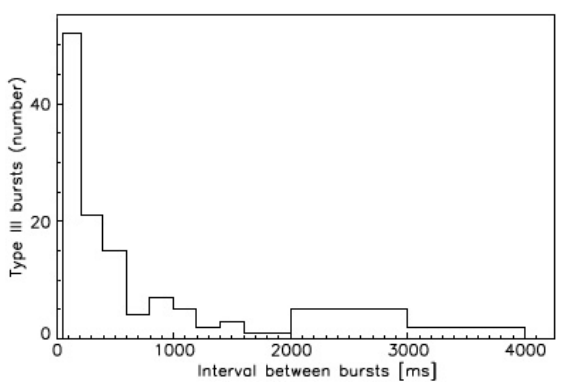

Fig. 8. Histogram of the distribution of time intervals between adjacent type III bursts.

\subsection{Time intervals between adjacent type III bursts}

The time interval between 120 adjacent drifting type III bursts (Table 2) is between 54 and $7013 \mathrm{~ms}$, with the average being $647 \pm 643 \mathrm{~ms}$. The average values of these intervals (Table 3 ) are similar for the entire frequency range. The bursts with small time intervals $(50-400 \mathrm{~ms})$ between adjacent bursts are the most frequent (Fig. 8).

\subsection{Groups of type III bursts with group frequency drift}

We found that 11 (of 17) groups (Table 4) show one or more group frequency drifts. Ten groups were detected at frequencies $f<2000 \mathrm{MHz}$, and only one group was found at higher frequencies. On average, there are 18 individual bursts per group. The mean group duration and bandwidth are $34 \pm 19 \mathrm{~s}$ and $339 \pm$ $160 \mathrm{MHz}$, respectively. Among a total of 25 group frequency drifts (Table 4), there are 13 positive and 12 negative ones, i.e. the numbers of the positive and the negative group drifts is almost identical. The positive group frequency drift is between 7 and $134 \mathrm{MHz} \mathrm{s}^{-1}$, with an average of $53 \pm 38 \mathrm{MHz} \mathrm{s}^{-1}$, while the negative group frequency drift is between -8 and $-170 \mathrm{MHz} \mathrm{s}^{-1}$, with average $-46 \pm 42 \mathrm{MHz} \mathrm{s}^{-1}$. These group frequency drifts are significantly smaller than the frequency drifts of individual bursts. In 9 of the 11 groups, there is more than one group frequency drift per group (Table 4). Examples of groups of type III bursts observed on September 21, 2000 (Fig. 9, left panel) and on September 20, 2000 (Fig. 9, right panel) are shown with negative $\left((1)\right.$ and $(2)=-9$ and $-20 \mathrm{MHz} \mathrm{s}^{-1}$ ) and positive $\left((3)\right.$ and $(4)=38$ and $\left.17 \mathrm{MHz} \mathrm{s}^{-1}\right)$ group frequency drifts, respectively. The bandwidths of the individual bursts are $62 \pm 20 \mathrm{MHz}$, and $49 \pm 7 \mathrm{MHz}$ on average, for the September 21, 2000 and September 20, 2000 groups, respectively.

Since 171 ( $85 \%$ of 201$)$ bursts are observed within the recorded frequency range and only 30 (15\% of 201) bursts escape the recorded range (Table 2, Part 1), the role of differently recorded frequency ranges is not so important in this case. Among a total of 201 bursts we determined 107 drifting bursts $(53 \%)$ and 94 bursts (47\%) with immeasurable drift. We found that among 107 drifting bursts, 49 bursts have positive 

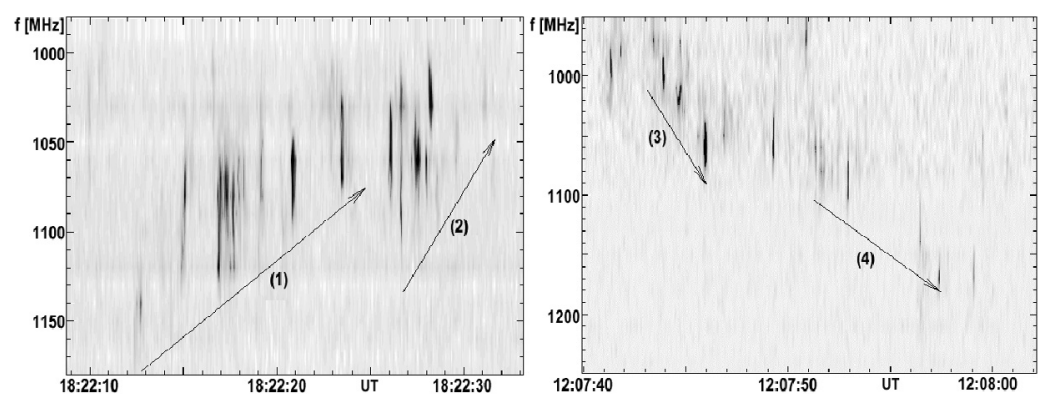

Fig. 9. Left: example of a group of type III bursts observed on September 21, 2000 with negative group frequency drifts (1) and (2) = -9 and $-20 \mathrm{MHz} \mathrm{s}^{-1}$, respectively. Right: example of a group of type III bursts observed on September 20, 2000 with positive group frequency drifts (3) and (4) $=38$ and $17 \mathrm{MHz} \mathrm{s}^{-1}$, respectively.

Table 4. Characteristic parameters of groups with frequency drift. Most of them have positive as well as negative $\operatorname{drift}(\mathrm{s})$.

\begin{tabular}{ccc}
\hline $\begin{array}{c}\text { Group } \\
\text { No. }\end{array}$ & $\begin{array}{c}\text { Positive group } \\
\text { frequency drift } \\
{\left[\mathrm{MHz} \mathrm{s}^{-1}\right]}\end{array}$ & $\begin{array}{c}\text { Negative group } \\
\text { frequency drift } \\
{\left[\mathrm{MHz} \mathrm{s}^{-1}\right]}\end{array}$ \\
\hline 2 & 97 & -170 \\
5 & 7 & -19 \\
6 & 19 & -12 \\
7 & 10 & -27 \\
8 & 38,17 & -9 \\
9 & $42,131,83,134$ & $-61,-61,-143$ \\
10 & 63,27 & $-9,-20$ \\
11 & & -8 \\
12 & 18 & -16 \\
14 & & -46 \\
17 & 53 & \\
\hline mean & &
\end{tabular}

drift rates that range from 98 to $2627 \mathrm{MHz} \mathrm{s}^{-1}$ (average $486 \pm$ $336 \mathrm{MHz} \mathrm{s}^{-1}$ ), and 58 bursts have negative drift rates that range from -92 to $-3232 \mathrm{MHz} \mathrm{s}^{-1}$ (average $-590 \pm 376 \mathrm{MHz} \mathrm{s}^{-1}$ ). The mean half-power duration and bandwidth of 100 drifting bursts within the recorded range are $106 \pm 43 \mathrm{~ms}$ and $105 \pm$ $51 \mathrm{MHz}$, respectively. The bandwidth of 71 bursts with immeasurable drift ranges from 21 to $189 \mathrm{MHz}$ (average $75 \pm 27 \mathrm{MHz}$ ).

\subsection{Groups of type III bursts without group frequency drift}

Apart from the bursts with group frequency drift, we found 6 groups that do not show group frequency drift. Three groups were detected at frequencies $f<2000 \mathrm{MHz}$ and three groups were detected at higher frequencies. On average, there are about 10 individual bursts per group i.e. there is a smaller number of bursts within a group than in the previous case of groups with group drift. The mean group duration of these groups is $17 \pm 9 \mathrm{~s}$.

Among a total of 61 type III bursts, 35 bursts (57\%) escape the recorded range and 26 bursts $(43 \%)$ are observed within the recorded frequency range (Table 2, Part 2). Among total 61 bursts, we determined 37 drifting bursts $(61 \%)$ and 24 bursts $(39 \%)$ with immeasurable drift. The mean half-power duration and bandwidth of 20 individual drifting bursts within the recorded range are $105 \pm 39 \mathrm{~ms}$ and $171 \pm 70 \mathrm{MHz}$, respectively. In this case, the role of different frequency ranges is important mainly due to the bandwidth of bursts. Since for these groups, the recorded range is 1000 or 500 or $200 \mathrm{MHz}$ (and most of these bursts are escaping the recorded range), we studied the bandwidth of the observed parts of these 35 escaping bursts (Table 2, Part 2). An example of a type III group without a group

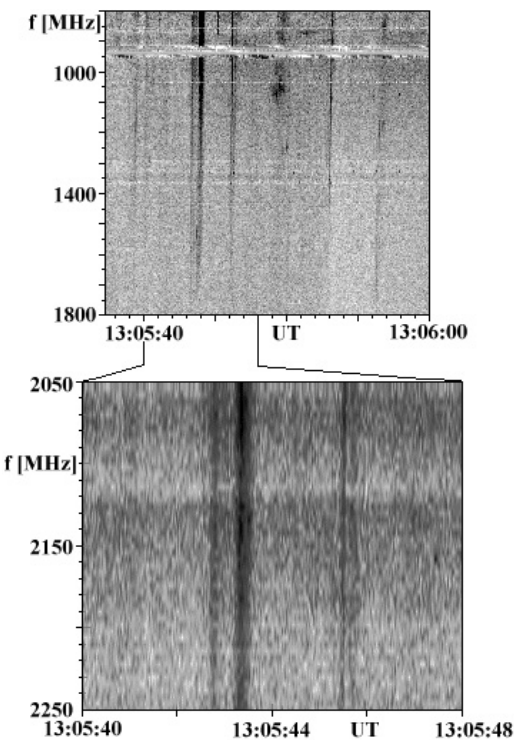

Fig. 10. Example of a group of type III bursts without group frequency drift, observed on September 13, 2001 by Ondřejov radiospectrometer (950-1800 MHz, top) and by the BSS (2050-2250 MHz, bottom).

drift and with escaping individual bursts, is shown in Fig. 10 (September 13, 2001), in the frequency range 950-2250 MHz, as observed by the BSS (bottom) in addition to the Ondřejov radiospectrometer (top). We found that among 37 drifting bursts, 15 bursts have a positive drift rate that ranges from 268 to $5528 \mathrm{MHz} \mathrm{s}^{-1}$, with an average $1857 \pm 1480 \mathrm{MHz} \mathrm{s}^{-1}$, and 22 bursts have a negative drift rate that ranges from -351 to $-4417 \mathrm{MHz} \mathrm{s}^{-1}$ (average $-1770 \pm 794 \mathrm{MHz} \mathrm{s}^{-1}$ ).

Considering the mean values, the bursts in groups without group drift are therefore less numerous (about 10 bursts), their group duration is shorter $(17 \mathrm{~s})$, individual bursts have higher bandwidth $(171 \mathrm{MHz})$ and significantly higher frequency drift $\left(+1857\right.$ and $\left.-1770 \mathrm{MHz} \mathrm{s}^{-1}\right)$, a higher number of bursts escape out of the recorded range (57\%), and a smaller number of bursts with immeasurable drift $(39 \%)$ with respect to the mean values which were found for the bursts in groups with group drift (18 bursts, 34 s, $105 \mathrm{MHz},+486$ and $-590 \mathrm{MHz} \mathrm{s}^{-1}, 15 \%, 47 \%$, respectively).

\section{Discussion and conclusions}

We investigated 17 groups of type III dm bursts, detected for the frequency range 950-2500 MHz, during 1999-2002, using the BSS. 
1) We analyzed 262 individual type III radio bursts and found 120 bursts with measurable frequency drift, within the recorded range, at the time of observation, as presented in Table 2. We found that most of these 120 bursts occur in the range 1100-1400 MHz. However, there are differences between the distributions of bursts with positive and negative frequency drift (Figs. 1 and 2). The half-power duration of most type III bursts is in the range of 50-150 ms. The mean value of this parameter decreases with increasing frequency and our measurements agree with the values in the range 100-600 ms, obtained by Meléndez et al. (1999), within the range 100-3000 MHz. The average value of the bandwidth of type III bursts with measurable frequency drift and bursts with immeasurable frequency drift is $116 \pm 67 \mathrm{MHz}$ and $77 \pm 28 \mathrm{MHz}$, respectively, and there is therefore a difference between them. The bandwidth is found to decrease with increasing frequency. Time intervals that occur between adjacent bursts are generally small (50-400 ms). We found that bursts with negative frequency drift (80) are more frequent than ones with positive frequency drift (64). The average drift of bursts with positive and negative drift is $807 \pm$ $675 \mathrm{MHz} \mathrm{s}^{-1}$ and $-914 \pm 684 \mathrm{MHz} \mathrm{s}^{-1}$, respectively. However, we recall that the number of bursts with immeasurable drift is rather high (118) and they are not involved in this evaluation. Most of the bursts with measurable frequency drift show values in the range $100-1000 \mathrm{MHz} \mathrm{s}^{-1}$. The frequency drift increases with increasing frequency. The high value of both uncertainty values is due to the high variance of individual drifts, i.e. there are bursts with very different drift values, which range from about 100 to more than $5000 \mathrm{MHz} \mathrm{s}^{-1}$.

Jiřička et al. (1993) detected type III bursts in the range 1.0-4.2 GHz that show generally negative drifts and only few of which have positive drift. In contrast we found that the positively-drifting bursts are not so rare. In another earlier study, Isliker \& Benz (1994) pointed out that positive drift rates of type III bursts, observed at $1-3 \mathrm{GHz}$ band, generally show a drift of $1 \mathrm{GHz} \mathrm{s}^{-1}$. This result is in a good agreement with our study.

Ma et al. (2006) investigated the statistics of 264 type III bursts observed in the $625-1500 \mathrm{MHz}$ range. They found the starting frequency of type III bursts to be about $700 \mathrm{MHz}$ and the frequency drift to be less than $1000 \mathrm{MHz} \mathrm{s}^{-1}$. Their statistical analysis, which is in good agreement with our present study, showed that the positive mean drift rate was $816 \mathrm{MHz} \mathrm{s}^{-1}$, while the negative mean drift rate was $-981 \mathrm{MHz} \mathrm{s}^{-1}$, in addition the average values of frequency drift increased with increasing frequency, while the average value of the half-power duration decreased with increasing frequency. There are however, some interesting differences. In Ma et al. (2006), the average values of the half-power duration and the bandwidth of the bursts were $791 \mathrm{~ms}$ and $289 \mathrm{MHz}$, while we measured $108 \mathrm{~ms}$ and $116 \mathrm{MHz}$ for measurable drift and $77 \mathrm{MHz}$ for immeasurable drift, respectively. In any way, the uncertainty limits are quite large. We observed a greater number of bursts with negative frequency drift than with positive drift, in contrast to Ma et al. (2006) who reported almost equivalent numbers. These differences are probably due to the fact that the observational frequency range $(625-1500 \mathrm{MHz})$ in $\mathrm{Ma}$ et al. (2006) is different (see the trend in Table 2).

2) We studied the distributions (Figs. 4, 5 and 7) of the half-power duration $H\left(f_{\text {start }}\right)$, bandwidth $B\left(f_{\text {start }}\right)$, and frequency drift $D\left(f_{\text {start }}\right)$, with respect to the starting frequency of a large number of type III bursts. These distributions were fitted by $F\left(f_{\text {start }}\right)=$ const. $f_{\text {start }}^{ \pm \alpha}$, where $F\left(f_{\text {start }}\right)$ represent $H\left(f_{\text {start }}\right)$ in $\mathrm{ms}$, or $B\left(f_{\text {start }}\right)$ in $\mathrm{MHz}$, or $D\left(f_{\text {start }}\right)$ in $\mathrm{MHz} \mathrm{s}^{-1} ; f_{\text {start }}$ is the starting frequency in $\mathrm{MHz}$, and $\alpha$ is the power-law index. If we use our relation $F\left(f_{\text {start }}\right)=$ const. $f_{\text {start }}^{ \pm \alpha}$, for $f_{\text {start }}=700 \mathrm{MHz}$, we find that $H\left(f_{\text {start }}\right)=378 \mathrm{~ms}, B_{1}\left(f_{\text {start }}\right)=289 \mathrm{MHz}$, and $D\left(f_{\text {start }}\right)=$ $391 \mathrm{MHz} \mathrm{s}^{-1}$. This result for $B_{1}\left(f_{\text {start }}\right)$ is similar to that reported by Ma et al. (2006). Differences in $H\left(f_{\text {start }}\right)$ and $D\left(f_{\text {start }}\right)$ are probably due to the different frequency range studied by Ma et al. (2006). Moreover, most bursts (201 of 262) in our work are bursts in groups with the group drift, which appears to be typical of decimetric type III bursts and different from metric ones.

3) We investigated the relations between various individual parameters of type III bursts (Fig. 11). Most bursts have small half-power durations $(<200 \mathrm{~ms}$, Fig. 11a), small bandwidths $(<200 \mathrm{MHz}$, Figs. 11a, c), small frequency drifts $\left(<2000 \mathrm{MHz} \mathrm{s}^{-1}\right.$ for positive and negative drift, Figs. 11b, d), and small time intervals between adjacent bursts $(<500 \mathrm{~ms}$, Figs. $11 \mathrm{~b}, \mathrm{c})$. The bursts with large bandwidths are observed to have longer half-power durations (Fig. 11a, see the trend in Table 3). The highest frequency drifts $\left(>2000 \mathrm{MHz} \mathrm{s}^{-1}\right.$ for positive and negative drift, Fig. 11d) occur in individual bursts with higher bandwidths $(>100 \mathrm{MHz})$, in contrast to the trend in Table 3, where the average values of 120 bursts are related mainly to drifting groups (100 bursts of 120 , Table 2$)$ i.e. to bursts in plasmoids (closed magnetic structures, see Fig. 12 and the following part 7). Individual bursts with high bandwidth in addition to the high frequency drift, are part of groups without drift (open magnetic structures, see the following part 7).

4) We found that 11 groups of type III bursts (for example in Fig. 9) show one or more group frequency drifts (on average $+53 \pm 38 \mathrm{MHz} \mathrm{s}^{-1}$ and $-46 \pm 42 \mathrm{MHz} \mathrm{s}^{-1}$ ), while in another 6 groups (example in Fig. 10), the group frequency drift cannot be determined. The high value of the uncertainty limits is due to the high variance of individual group drifts (see Table 4). This variance in both the positive and negative group drift can be interpreted as a variance in the plasmoid velocity (see Fig. 12 and the following part 7).

While bursts without group drift are often observed with starting or ending frequency that is beyond the recorded frequency range of the instrument (57\% of total 61 bursts), bursts in drifting groups are usually observable within the recorded range (85\% of total 201 bursts). It appears that type III bursts in groups with group drift are similar to a DPS (drifting pulsating structure; Karlický et al. 2002; 2005). Thus, these type III bursts can also have their origin in closed magnetic structures (plasmoids). These groups of type III bursts contain on average more individual bursts (18), last longer (34 s), and have bursts of smaller bandwidth (105 MHz) than groups without group drift (on average 10 bursts, $17 \mathrm{~s}$ and $171 \mathrm{MHz}$ ), respectively. The groups of bursts without group drift are similar to the type III bursts observed in the metre-wavelength range where they evolve in open magnetic structures. The average positive and negative frequency drift of individual bursts are significantly different for these two group types: $\left(486 \pm 336\right.$ and $\left.-590 \pm 376 \mathrm{MHz} \mathrm{s}^{-1}\right)$ and $\left(1857 \pm 1480\right.$ and $\left.-1770 \pm 794 \mathrm{MHz} \mathrm{s}^{-1}\right)$ for bursts with and without group drift, respectively. Type III bursts that belong to the same drifting group have the same characteristic bandwidth. For example, the bandwidth of the May 08, 2000 bursts ranges from 47 to $90 \mathrm{MHz}$ (on average $64 \mathrm{MHz}$ ), while the bandwidth of the Jun. 28, 1999 bursts ranges from 70 to $155 \mathrm{MHz}$ (on average $105 \mathrm{MHz}$ ). Thus, the characteristic bandwidth of bursts in the drifting group can reflect the size of the plasmoid (see Fig. 12 and the following part 7).

5) As an example, we computed the electron density, the coronal altitude and the velocity of such up/down moving plasmoids for the September 21, 2000 groups in Fig. 9, left panel, groups (1) and (2) as well as for the September 20, 2000 groups 

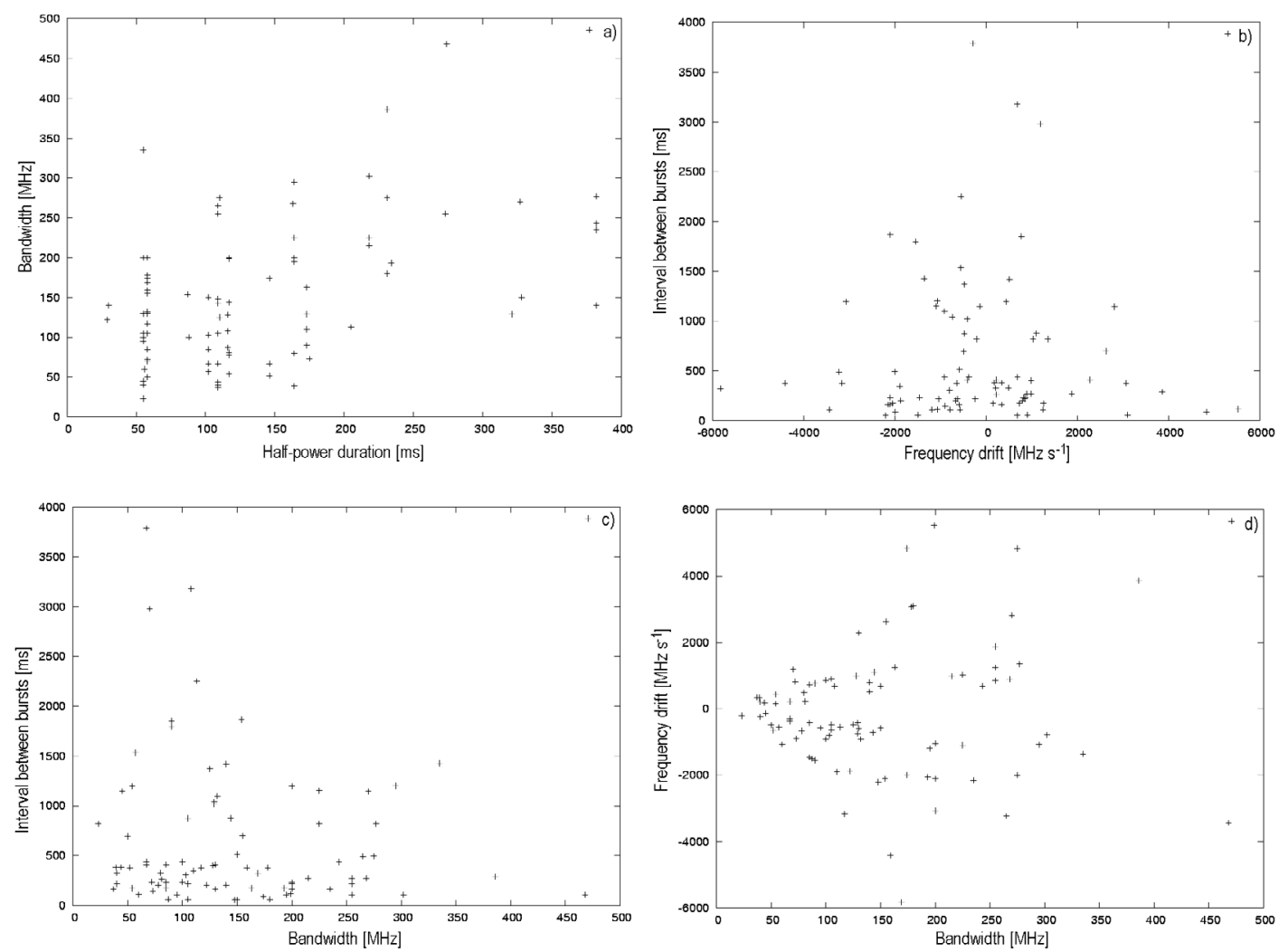

Fig. 11. Relationship between various parameters: a) bandwidth vs. half-power duration, b) intervals between adjacent bursts vs. drift of bursts, c) intervals between adjacent bursts vs. bandwidth, d) drift of bursts vs. bandwidth. The crosses indicate individual bursts. (The concentration of bursts at about $50 \mathrm{~ms}$ in a) is due to the $50 \mathrm{~ms}$ time-resolution of the instrument.)

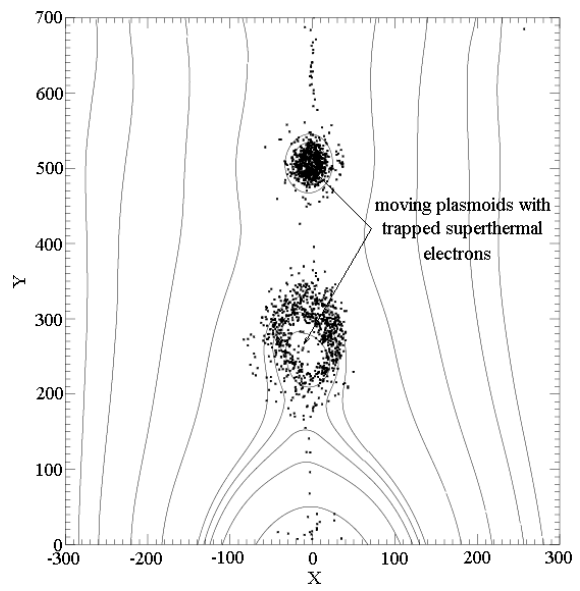

Fig. 12. Distribution of accelerated electrons (points) (with the velocity $|v|>0.55 \mathrm{c}$, where $c$ is the speed of light) in the plasmoids above the flare arcade structure at $\omega_{\text {pe }} t=250 ; x$ - and $y$-coordinate represent the horizontal distance and height in the solar atmosphere, respectively. The full lines are the projections of the magnetic-field lines onto the $x-y$ plane. The scale unit is the grid distance.

in Fig. 9, right panel, groups (3) and (4). We used the density model of Aschwanden \& Benz (1995) with numerical values of height $h_{1}=1.6 \times 10^{2} \mathrm{Mm}$, density of the quiet corona $n_{\mathrm{Q}}=$ $4.6 \times 10^{8} \mathrm{~cm}^{-3}$, and the parameter $p=2.38$. Thus, we obtain the average electron densities $n_{\mathrm{e}}=1.6 \times 10^{10}, 1.4 \times 10^{10}, 1.3 \times$ $10^{10}$, and $1.6 \times 10^{10} \mathrm{~cm}^{-3}$, at mean coronal altitudes $h_{0}=13.3$, 14.0, 14.4, and 13.3 Mm, with velocities of moving plasmoids $v_{\mathrm{p}}=81,221,447$, and $164 \mathrm{~km} \mathrm{~s}^{-1}$, for the groups (1)-(4), respectively. We measured both negative and positive group drifts
(Table 4, Fig. 9) due to radio emission from up or down moving plasmoids in flaring current sheets (see Fig. 12 and the following part 7).

6) It is interesting that most of the type III burst groups (6 of 10) occur in the declining phase of the corresponding GOES flare and only four type III burst groups occur during its impulsive phase. We showed that there are no significant differences between properties of type III burst groups in the impulsive and in the declining phase of a flare. Thus, the process of the creation and the tearing of the plasmoids starts during the impulsive phase. Individual plasmoids are then moving up as well as down (have negative as well as positive group frequency drift) in the solar atmosphere. This process can also occur during the declining phase of the flares.

7) To illustrate the trapping of superthermal electrons in plasmoids and show why most dm type III bursts are limited to some frequency ranges, we present, in Fig. 12, the results of our recent numerical simulations. In a manner similar to that reported by Karlický \& Bárta (2006), we first simulated a 2D cusp magneticfield structure, which represented a magnetic-field topology in flares. Then, we placed the magnetic field into the $2.5 \mathrm{D}$ relativistic electromagnetic code (for the code, see Karlický \& Bárta 2007). The system size was $L_{x}=600 \Delta$ and $L_{y}=2000 \Delta$, where $\Delta(=1)$ is the grid size (where the Debye length is $0.5 \Delta$ ). Thus, the real system size is small. Nevertheless, we believe that the physical processes will be similar to those in much larger systems. In each numerical cell, we started with 60 electrons and 60 protons. The guiding magnetic field, which was perpendicular to the computational box, was $B_{z}=0.3 \times B_{0}$, where $B_{0}$ is the asymptotic value of $\left|B_{y}\right|$ for large $x$. The plasma beta parameter and the ratio of the electron-cyclotron and electron-plasma frequencies in the region outside the current sheets, were chosen 
to be $\beta \sim 0.02$ and $\omega_{\text {ce }} / \omega_{\text {pe }}=0.45$, respectively. The electron thermal velocity was taken to be the same inside the entire numerical box, since $v_{\mathrm{Te}}=0.05 c$, where $c$ is the speed of light. The proton-electron mass ratio $m_{\mathrm{p}} / m_{\mathrm{e}}=16$ was used. This number is not realistic, but was assumed so as to shorten considerably the computational time. Nevertheless, the electron mass is sufficiently low to be able to separate accurately the dynamics of electrons and protons.

As can be seen in Fig. 12, the plasmoids are formed due to the tearing mode-instability in the current sheet above the magnetic field arcade. As found in Karlický \& Bárta (2007) the most energetic electrons are accelerated close to the X-points of the magnetic field structure during this process. They are also accelerated by pinching of the plasmoids. Thus, as shown in Fig. 12, superthermal electrons are trapped in plasmoids (semi-closed magnetic-field structures) or some escape along open magneticfield lines. All electrons can generate Langmuir waves, either by the bump-on-tail, or by some other instabilities, which are then converted to electromagnetic waves. Their frequency corresponds to the local plasma frequency or its harmonic. If we assume that the density decreases with height in the solar atmosphere, which is not considered in the numerical model, then the radio spectrum of those processes show two categories of type III bursts: a) groups of type III bursts which are generated by superthermal electrons trapped in plasmoids and drift as a whole with a frequency drift that correspond to the plasmoid velocity, and b) type III bursts generated by electron beams moving along open magnetic-field lines. This division corresponds well to that found in the statistical analysis of the observed bursts.

Acknowledgements. The authors thank the unknown referee and Prof. P. Janardhan for their useful remarks that improved this paper. H.M. acknowledges the FAPESP support for the project 2006/50039-7.
M.K. acknowledges the support from the Grant IAA300030701 of the Academy of Sciences of the Czech Republic. F.C.R.F. thanks CNPq for scholarship (proc. 310005/2005-1). J.R.C. acknowledges the CNPq support for project 475723/2004-0.

\section{References}

Aschwanden, M. J. 2002, Space Sci. Rev., 101, 1

Aschwanden, M. J., \& Benz, A. O. 1995, ApJ, 438, 997

Bárta, M., \& Karlický, M. 2005, ApJ, 631, 612

Benz, A. O. 2004, in Solar and Space Weather Radiophysics, The Netherlands, ed. D. E. Gary, \& C. U. Keller, 203

Cecatto, J. R., Sawant, H. S., Fernandes, F. C. R., et al. 2003, Adv. Space Res., 32,2533

Isliker, H., \& Benz, A. O. 1994, Acta Astrophys. Sin., 104, 145

Jiřička, K., Karlický, M., Kepka, O., \& Tlamicha, A. 1993, Sol. Phys., 147, 203

Karlický, M., \& Bárta, M. 2006, ApJ, 647, 1472

Karlický, M., \& Bárta, M. 2007, A\&A, 464, 735

Karlický, M., Fárník, F., \& Mészárosová, H. 2002, A\&A, 395, 677

Karlický, M., Bárta, M., Mészárosová, H., \& Zlobec, P. 2005, A\&A, 432, 705

Kundu, M. R. 1998, ESA SP-421, 179

Li, K. J., Gu, X. M., \& Chen, X. K. 2000, MNRAS, 313, 761

Lin, J., Soon, W., \& Baliunas, S. L. 2003, New Astron. Rev., 47, 53

Ma, Y., Wang, S. L., Yan, Y. H., \& Fu, Q. J. 2002, Astrophys. Space Sci., 279, 219

Ma, Y., Xie, R. X., Zheng, X. M., et al. 2005, IAU Symp., 226, 143

Ma, Y., Xie, R. X., \& Wang, M. 2006, Sol. Phys., 238, 105

Meléndez, J. L., Sawant, H. S., Fernandes, F. C. R., \& Benz, A. O. 1999, Sol. Phys., 187, 77

Moore, R., McKenzie, D. L., Svestka, Z., et al. 1980, in Solar Flares: monograph from Skylab Solar Workshop II, Report of NASA Skylab Workshop on Solar Flares, Boulder, ed. P. A. Sturrock (Colorado University Press), 341

Ohyama, M., \& Shibata, K. 1998, ApJ, 499, 934

Poquérusse, M., Hoang, S., Bougeret, J. L., \& Moncuquet, M. 1995, Solar Wind 8 Proc., Dana Point/California, June 1995, 62

Sawant, H. S., Fernandes, F. C. R., \& Neri, J. A. C. F. 1994, ApJS, 90, 689 Sawant, H. S., Subramanian, K. R., Faria, C., et al. 2001, Sol. Phys., 200, 167

Tanuma, S., \& Shibata, K. 2005, ApJ, 628, L77 